\title{
R127 AND ITS SURROUNDING STAR CLUSTER
}

\author{
ANTONELLA NOTA ${ }^{1,2}$, CLAUS LEITHERER ${ }^{1,3}$, MARK CLAMPIN $^{4}$, and \\ ROBERTO GILMOZZI ${ }^{1,3}$ \\ ${ }^{1}$ STScI, 3700 San Martin Drive, Baltimore, MD 21218 \\ 2 On leave from the Observatory of Padova, Italy \\ ${ }^{3}$ Affiliated with the Astrophysics Division, Space Science Department of ESA \\ 4 Johns Hopkins University, Homewood Campus, Baltimore, MD 21218
}

The LMC star R127 (=HDE 269858) has originally been classified as a late WN type (Walborn 1977). Since then, R127 has developed from spectral type B to A (Wolf et al. 1988) and is now categorized as a Luminous Blue Variable. R127 is embedded in gaseous material and a star cluster which are barely resolvable even on the highest-quality images available. Stahl (1987) studied the circumstellar material using narrow-band CCD images centered on nebular emission lines. No systematic census of the stars in the immediate vicinity of R127 has yet been published.

We obtained high-resolution images of R127 with the STScI coronograph mounted on the ESO/MPI 2.2-m telescope in February 1989 (see Paresce, Burrows, and Horne 1987 for a description of the instrument). R127 is occulted by a long, thin wedge in order to reduce its brightness $\left(V \approx 9^{m}\right)$ relative to the surrounding stars. Figure 1 is a $V$ image of the region around R127. (Field size: 22" $\times 36$ "; plate scale: 0.14 " per pixel; FWHM of stellar images: 0.9 "). R127 is surrounded by a number fainter stars, most of which were previously unknown due to the brightness of R127 itself. V photometry of the stars has been obtained by calibrating the $\mathrm{V}$ frame with the standard star Feige 15 (see Table 1 ). No color transformations have been performed. The photometric errors are less than 0.2 .

R127 has a companion (designated R127B) with $V=12.9$ at a distance of $~ 3.5$ " to the northwest. Another close companion (R127U, $V=16.1$ ) is 3.6" to the south. Although these companions make a negligible contribution to the visual flux of R127, their influence in the UV at $\sim 1500 \AA$ may be crucial if observations with insufficient resolution are performed. Stahl et. al (1983) report that IUE spectra of R127 are contaminated by R127B and another companion (R127U?). Since the UV flux of R127 has decreased substantially in the meantime, the light contribution in the UV of these companions will be much more important at present.

All stars in the field of view are most probably LMC stars which are not physically associated with R127.

Our high-resolution image gives no indication for multiplicity of R127. The point-spread function is consistent with the assumption that only one star significantly contributes to the visual light. Our results support the suggestion that $R 127$ is a very luminous $\left(L \approx 10^{6} L_{\odot}\right)$ star with an initial mass between $60 M_{\odot}$ and $100 M_{\odot}$.

K. A. van der Hucht and B. Hidayat (eds.),

Wolf-Rayet Stars and Interrelations with Other Massive Stars in Galaxies, 561-562.

(c) 1991 IAU. Printed in the Netherlands. 


\section{References}

Paresce, F., Burrows, C., and Horne, K. 1988, Ap. J., 329, 318.

Stahl, O. 1987, Astr. Ap., 182, 229.

Stahl, O., Wolf, B., Klare, G., Cassatella, A., Krautter, J., Persi, P., and

Ferrari-Toniolo 1983, Astr. Ap., 127, 49.

Walborn, N. R. 1977, Ap. J., 215, 53.

Wolf, B., Stahl, O., Smolinski, J., and Cassatella, A. 1988, Astr. Ap. Suppl., 74, 239.
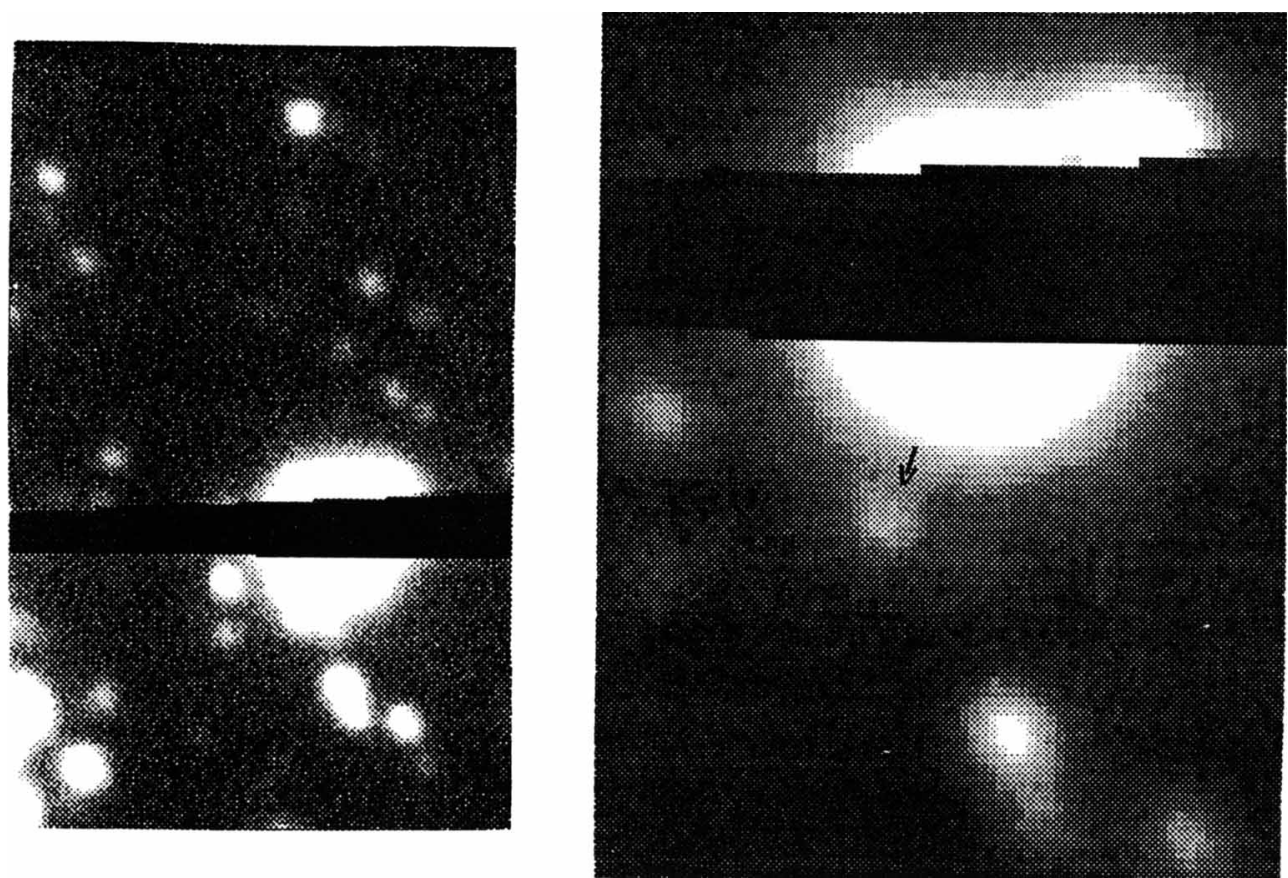

Figure 1. Coronographic V image of the field around R127. The right-hand figure is an enlarged version of the left-hand figure. North is up and east to the left. R127 itself is partly occulted by the wedge. The position of R127U is indicated by an arrow.

\begin{tabular}{||c|r|r|c||}
\hline Star & x-Pos & y-Pos & V \\
\hline B & 109 & 117 & 12.9 \\
C & 107 & 155 & 18.2 \\
D & 131 & 166 & 18.3 \\
E & 123 & 141 & 17.6 \\
F & 133 & 134 & 18.0 \\
G & 81 & 166 & 19.0 \\
H & 13 & 208 & 16.8 \\
I & 23 & 183 & 17.5 \\
J & 95 & 227 & 16.0 \\
K & 114 & 176 & 17.4 \\
\hline
\end{tabular}

\begin{tabular}{||c|r|r|c||}
\hline Star & x-Pos & y-Pos & V \\
\hline $\mathrm{L}$ & 34 & 119 & 17.5 \\
$\mathrm{M}$ & 69 & 79 & 15.9 \\
$\mathrm{~N}$ & 69 & 61 & 17.2 \\
$\mathrm{O}_{2}$ & 107 & 42 & 14.8 \\
$\mathrm{P}$ & 126 & 34 & 17.0 \\
$\mathrm{Q}$ & 134 & 22 & 17.9 \\
$\mathrm{R}$ & 61 & 28 & 18.8 \\
$\mathrm{~S}$ & 31 & 40 & 16.8 \\
$\mathrm{~T}$ & 25 & 19 & 15.0 \\
$\mathrm{U}$ & 94 & 67 & 16.1 \\
\hline
\end{tabular}

Table 1. Photometry of the R127 cluster. The zero point of the coordinate system is in the southeast corner of Figure 1. The northwest corner has $x=160, y=$ 256. 